\title{
Small-angle neutron scattering study of aggregate structures of multi-headed pyridinium surfactants in aqueous solution
}

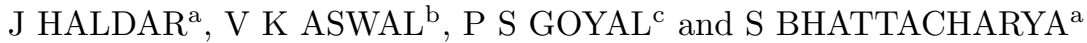 \\ ${ }^{a}$ Department of Organic Chemistry, Indian Institute of Science, Bangalore 560 012, India \\ ${ }^{\mathrm{b}}$ Solid State Physics Division; ${ }^{\mathrm{c}}$ IUC-DAEF, Mumbai Centre, Bhabha Atomic Research \\ Centre, Mumbai 400 085, India \\ E-mail: jaya@students.orgchem.iisc.ernet.in
}

\begin{abstract}
The aggregate structures of a set of novel single-chain surfactants bearing one, two and three pyridinium headgroups have been studied using small-angle neutron scattering (SANS). It is found that the nature of aggregate structures of these cationic surfactants depend on the number of headgroups present in the surfactants. The single-headed pyridinium surfactant forms the lamellar structure, whereas surfactants with double and triple headgroups form micelles in water. The aggregates shrink in size with increase in the number of headgroups in the surfactants. The aggregation number $(N)$ continually decreases and the fractional charge $(\alpha)$ increases with more number of headgroups on the surfactants. The semimajor axis $(a)$ and semiminor axis $(b=c)$ of the micelle also decrease with the increase in the number of headgroups in the surfactants. This indicates that hydrocarbon chains in such micelles prepared from multiheaded surfactants adopt bent conformation and no longer stay in extended conformation.
\end{abstract}

Keywords. Multi-headed pyridinium surfactants; micelle; small-angle neutron scattering.

\section{PACS Nos 61.12.Ex; 82.70.Uv}

\section{Introduction}

Micelles are formed by the aggregation of the surfactants in their aqueous solution above a certain concentration known as the critical micellar concentration (CMC) [1]. The hydrophobic segments of the surfactant molecules constitute the interior core of the micelles to avoid exposure to water and the polar headgroups reside at the micellar exterior interfacing with the bulk water. Various types of surfactants that vary in terms of their headgroup charges or in other parts of their molecular architecture are known. Even among cationic surfactants several variations in their molecular structures are known. For instance cationic surfactants consisting of single-chain, single-headgroup e.g. cetyltrimethylammonium bromide (CTAB) or cetylpyridinium bromide (CPB) are known, the micellar properties of which have been extensively investigated [2]. Clearly the differences between trimethy- 
lammonium and pyridinium headgroups, the charge distribution and the hydration properties of the cations influence the variation in their micellar properties. While it has been shown that single-chain surfactants bearing one, two and even three trimethylammonium bromide headgroups form micelles [3], nothing is known about the corresponding series of surfactants bearing pyridinium headgroups. Herein, we present aggregate structures of three new single-chain surfactants possessing one, two or three pyridinium headgroups (figure 1) and also compare their structures with that of CPB. Small-angle neutron scattering (SANS) measurements have been carried out to determine the aggregate structures in these surfactant solutions.

\section{Experiment}

Synthesis. The compounds $\mathbf{1}(h=1), \mathbf{2}(h=2)$ and $\mathbf{3}(h=3)$ were synthesized via esterification of 1-hexadecanol, 2-hydroxymethyl-hexadecanol or 2,2dihydroxymethyl-hexadecanol respectively with $1.1,2.2$ and 3.3 equiv. of 2 bromoacetyl bromide in the presence of $\mathrm{Et}_{3} \mathrm{~N}$ and a catalytic amount of DMAP in $\mathrm{CH}_{2} \mathrm{Cl}_{2}$. After purification, the respective bromoacetylated products were quaternized with pyridine in dry acetone at $50^{\circ} \mathrm{C}$ in a screw-top pressure tube. Pure surfactants 1 and $\mathbf{2}$ were obtained upon repeated recrystallizations from $\mathrm{CHCl}_{3} / n$ hexane and $\mathrm{CHCl}_{3} /$ acetone $/ n$-hexane respectively. Surfactant 3 was purified by repetitive precipitations from $\mathrm{CHCl}_{3} / n$-hexane mixture.

SANS measurements. Solubilization of the newly synthesized surfactants in water was achieved depending on the number of headgroups that these surfactants possessed. The solubilization of single-headed surfactant $(h=1)$ required heating to obtain the transparent solution. Therefore all the experiments were performed at $40^{\circ} \mathrm{C}$. Surfactants with $h=2$ and $h=3$ were however, highly soluble in $\mathrm{H}_{2} \mathrm{O}$ at ambient temperature and the solutions were transparent. We believe that the facile solubilization in water was achieved by increasing the hydrophilicity of the surfactant with the increase in the number of headgroups. All the solutions used in the SANS experiments were prepared in $\mathrm{D}_{2} \mathrm{O}$ in place of $\mathrm{H}_{2} \mathrm{O}$ because this provides good contrast between the micelles and the solvent. SANS experiments were performed using SANS diffractometer at the Swiss Spallation Neutron Source SINQ, Paul Scherrer Institute [4]. The wavelength of the neutron beam was $8 \AA$ and the experiments were performed at two different sample-to-detector distances of 2 and $6 \mathrm{~m}$ to cover a $Q$ range of 0.008 to $0.4 \AA^{-1}$. The scatter neutrons were detected using a two-dimensional $96 \mathrm{~cm} \times 96 \mathrm{~cm}$ detector. The solutions were held in $1 \mathrm{~mm}$ path length UV grade quartz sample holders with tight fitting teflon stoppers. The measured data have been corrected and normalized to a cross-section unit, using standard procedure.

\section{SANS analysis}

In small-angle neutron scattering one measures the differential scattering crosssection per unit volume $(\mathrm{d} \Sigma / \mathrm{d} \Omega)$ as a function of scattering vector $Q$. For a miceller solution, $\mathrm{d} \Sigma / \mathrm{d} \Omega$ can be expressed as [3] 


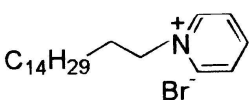

CPB

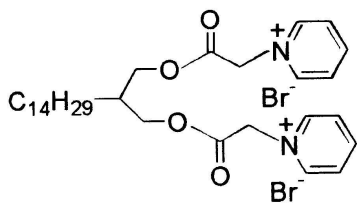

$2(h=2)$

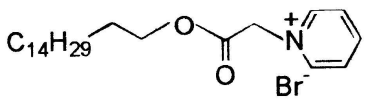

$1(h=1)$

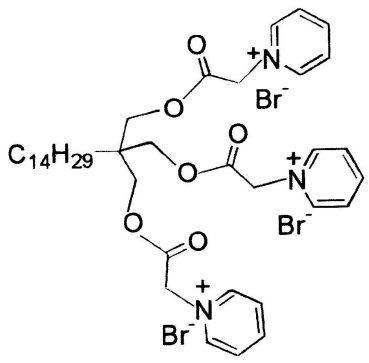

$3(h=3)$

Figure 1. Chemical structure of multi-headed pyridinium surfactants.

$$
\frac{\mathrm{d} \Sigma}{\mathrm{d} \Omega}(Q)=n\left(\rho_{\mathrm{m}}-\rho_{\mathrm{s}}\right)^{2} V^{2} P(Q) S(Q),
$$

where $n$ is the number density of the micelles, $\rho_{\mathrm{m}}$ and $\rho_{\mathrm{s}}$, are respectively, the scattering length densities of the micelle and the solvent and $V$ is the volume of the micelle. $P(Q)$ is the intraparticle structure factor and depends on the shape and size of the particle. $S(Q)$ is the interparticle structure factor and is decided by the interparticle distance and the particle interactions. We have used $P(Q)$ for ellipsoidal micelles and $S(Q)$ has been calculated using the method of Hayter and Penfold, which assumes the screened Coulomb interaction between the charged micelles [5]. The dimensions of the micelles, aggregation number and fractional charge have been determined from the analysis. The semimajor axis $(a)$, semiminor axis $(b=c)$ and the fractional charge $(\alpha=Z / N$, where $Z$ is the micellar charge $)$ are the parameters in analysing the SANS data. The aggregation number is calculated by the relation $N=4 \pi a b^{2} / 3 v$, where $v$ is the volume of the surfactant monomer.

\section{Results and discussion}

Figure 2 shows the SANS data from aqueous solution of single-, double- and tripleheaded surfactants along with that of CPB at fixed surfactant concentration (25 $\mathrm{mM}$ ) and at $40^{\circ} \mathrm{C}$. SANS data of double-, triple-headed surfactants and CPB show correlation peak as expected from suspension of charged particles such as cationic micelles. However, in the case of single-headed surfactant $(h=1)$, the characteristic of SANS data does not suggest that this system also contains the micelles. In the case of micellar solutions SANS peak occurs at $Q_{\mathrm{m}} \approx 2 \pi / d$, where $d$ is the average distance between micelles. Since $Q_{\mathrm{m}}$ was found to vary with the number of headgroups $(h)$, one can conclude that intermicelle distance and hence the number density $(n)$ of micelles is different for CPB, double- and triple-headed surfactant solutions even when they have identical surfactant concentration. This suggests that 


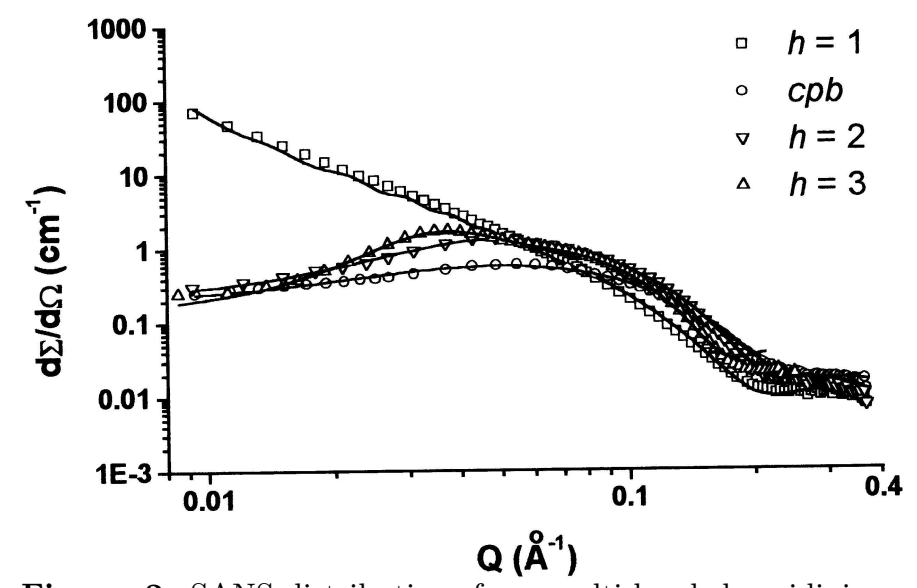

Figure 2. SANS distributions from multi-headed pyridinium surfactants at $25 \mathrm{mM}$ and $40^{\circ} \mathrm{C}$.

the aggregation number $(N)$ of the micelle vary with the number of headgroups $(h)$. The detailed analysis further confirms that micelles are indeed formed from $\mathrm{CPB}$, double- and triple-headed surfactants irrespective of the number of headgroups $(h)$. It is found that the aggregation number $(N)$ decreases from $122(\mathrm{CPB})$ to $55(h=2)$ and then to $32(h=3)$ with increase in the number of headgroups per single hydrocarbon chain (table 1). These results are understood as larger headgroup size and enhanced electrostatic repulsion with $h=2$ and $h=3$ will require greater micellar surface and would only be able to accommodate fewer number of surfactant molecules to pack into a single micellar aggregate. The fractional charge $(\alpha)$ on the micelle increases from $0.18(\mathrm{CPB})$ to $0.20(h=3)$ with the number of headgroups on the surfactant. Both the semimajor $(a)$ and semiminor axes $(b=c)$ decrease with increase in the number of headgroups. This is expected as with every increase in the number of cationic headgroup the charge repulsion at the headgroup level among the proximal surfactant molecule become more and more pronounced. To alleviate these unfavorable electrostatic repulsions, the hydrocarbon chains in the micelles of the multiple-headed surfactants most likely adopt folded conformations leading to the formation of increasingly disordered collection of surfactant molecules in the resulting micellar aggregate.

The higher scattering intensity and the non-existence of correlation peak in the case of $h=1$ indicate the formation of large structure with this system. It is found that the scattering intensity in the low- $Q$ region $\left(<0.08 \AA^{-1}\right)$ decreases as $1 / Q^{2}$

Table 1. Micellar parameters of multi-headed surfactants.

\begin{tabular}{lrccc}
\hline Surfactant & $N$ & $\alpha$ & $b=c(\AA)$ & $a(\AA)$ \\
\hline CPB & 122 & 0.18 & 24.0 & 29.9 \\
$h=2$ & 55 & 0.19 & 19.0 & 34.2 \\
$h=3$ & 32 & 0.20 & 16.5 & 32.1 \\
\hline
\end{tabular}


is almost in a straight line and this suggests the formation of either lamellar or vesicular structure for $h=1[6]$. At higher $Q$ values $\left(>0.08 \AA^{-1}\right)$, intensity decreases and a minimum is observed. The transmission electron micrograph (TEM) measurement on this system confirms the presence of lamellar structure of width 40-50 nm and a length of $\sim 2 \mu \mathrm{m}$. We have, therefore, fitted the data for $h=1$ assuming the lamellar structure, where the intensity varies as $1 / Q^{2}$ in the low $Q$ region and the large $Q$ data gives the thickness of the lamellar structure. The fitted value of thickness is about $30 \AA$.

While CPB forms micellar aggregate in water, $h=1$ forms lamellar structure even though both have single headgroup per hydrocarbon chain. It is possible that surfactant $h=1$ is more tightly associated in the aggregate because of the presence of an ester linkage $[-\mathrm{OC}(\mathrm{O})-]$ between the pyridinium headgroup and the hydrocarbon chain. The presence of this linkage facilitates intermonomer association through dipolar interactions. These ester linkages are located near the 'stern layer' region of the micelles [7], strong hydrogen bonding interactions among the surfactant molecules can occur through interfacially associated water molecules [8]. Furthermore, unlike surfactants with trimethylammonium headgroups, surfactants with pyridinium headgroups will be having extra aromatic $\pi-\pi$ interactions between the headgroups and that might also bring the surfactant monomers together resulting in the formation of lamellar structure.

In summary, we have studied aggregation properties of the newly synthesized surfactants with multiple headgroups. The results suggest that aggregation properties could be remarkably altered by the number of headgroups on the surfactant. The aggregate size decreases drastically with increase in the number of headgroups in the surfactants. However, at this stage the maximum number of charged headgroups that can be attached onto a single hydrocarbon chain till micelle formation is feasible remains to be examined.

\section{References}

[1] F M Menger, Angew. Chem. Int. Ed. Engl. 30, 1086 (1991) R Zana and Y Talmon, Nature 362, 228 (1993)

[2] T Perche, X Auvray, C Petipas, R Anthore, S Meliani, E Perez, I Rico and A Lattes, J. de Phys. IV: Proceedings 3, 133 (1993)

[3] J Haldar, V K Aswal, P S Goyal and S Bhattacharya, Angew. Chem. Int. Ed. 40, 1228 (2001)

J Haldar, V K Aswal, P S Goyal and S Bhattacharya, J. Phys. Chem. 105, 12803 (2001)

[4] J Kohlbrecher and W Wagner, J. Appl. Crystallogr. 33, 804 (2000)

[5] J B Hayter and J Penfold, Colloid Polym. Sci. 21, 751 (1988)

[6] V K Aswal, S De, P S Goyal, S Bhattacharya and R K Heenan, Phys. Rev. E57, 776 (1998) V K Aswal, J Haldar, S De, P S Goyal and S Bhattacharya, Phys. Chem. Chem. Phys. 5, 907 (2003)

[7] J H Fendler, Membrane mimetic chemistry (John Wiley \& Sons Inc., New York, 1982)

[8] R A Moss, S Ganguli, Y Okumura and T Fujita, J. Am. Chem. Soc. 11, 6391 (1990) and references therein S Bhattacharya and S Haldar, Langmuir 11, 4748 (1995) 